\title{
PERCEPCIONES SOBRE EL APRENDIZAJE VIRTUAL CON MICROLEARNING: ESTUDIO DE CASO DE UNA EXPERIENCIA DE FORMACIÓN PROFESIONAL EN UNA ORGANIZACIÓN PRIVADA PERCEPTIONS ABOUT VIRTUAL LEARNING WITH MICROLEARNING: CASE STUDY OF A PROFESSIONAL TRAINING EXPERIENCE IN A PRIVATE ORGANIZATION
}

\section{Carol Rivero Panaqué}

Edith Soria-Valencia ${ }^{2}$

Pontificia Universidad Católica del Perú

\section{RESUMEN}

El presente artículo analiza las características, beneficios, inconvenientes y efectividad del

$1 \quad$ Docente del Departamento de Educación de la Pontificia Universidad Católica del Perú. Directora de la Maestría en Integración e Innovación Educativa de las Tecnologías de la Información y la Comunicación (TIC). Miembro del Grupo de investigación EDUTEC-PUCP [https://orcid.org/0000-0002-0123-8999] crivero@pucp.edu. pe

2 Docente del Departamento de Educación de la Pontificia Universidad Católica del Perú. Coordinadora de la Maestría en Integración e Innovación Educativa de las Tecnologías de la Información y la Comunicación (TIC). Miembro del Grupo de investigación EDUTEC-PUCP. [https://orcid.org/0000-0002-2249-7826] edith.soria@pucp. edu.pe microlearning a partir de una experiencia de formación virtual brindada a los colaboradores de una empresa privada de seguros. Los objetivos del estudio son: a) identificar las características del microlearning en los cursos de formación virtual brindados a los colaboradores de una empresa, b) describir los beneficios e inconvenientes que genera el microlearning, c) identificar en las cápsulas de microcontenido los elementos significativos que permitieron el aprendizaje. La investigación presenta un diseño descriptivo a partir de un estudio de caso y las técnicas de recojo de información fueron la encuesta y el grupo focal. 
En la investigación participan 133 profesionales de diversas disciplinas que laboran en diferentes puestos de trabajo en una empresa privada de seguros. Los resultados muestran que el éxito del microlearning está asociado al diseño de programas de formación profesional que consideren las edades, necesidades y demandas de los usuarios, quienes evidentemente tienen requerimientos de aprendizaje diferentes.

\section{PALABRAS CLAVE}

Aprendizaje virtual, microaprendizaje, formación profesional, aprendizaje de adultos, aprendizaje permanente.

\section{ABSTRACT}

This article analyzes the characteristics, benefits, drawbacks, and effectiveness of microlearning from a virtual training experience provided to employees of a private insurance company. The objectives of the study are: a) to identify the characteristics of microlearning in the virtual training courses offered to the collaborators of a company, b) to describe the benefits and drawbacks generated by microlearning, c) to identify the significant elements in the microcontent capsules that allowed learning. The research presents a descriptive design based on a case study and the information gathering techniques were the survey and the focus group. 133 professionals from various disciplines who work in different jobs in a private insurance company participate in the research. The results show that the success of microlearning is associated with the design of professional training programs that consider the ages, needs and demands of the users, who obviously have different learning requirements.

\section{KEYWORDS}

Virtual learning, microlearning, vocational training, adult learning, lifelong learning.

\section{PERCEPÇÕES SOBRE A APRENDIZAGEM VIRTUAL COM MICROLEARNING: ESTUDO DE CASO DE UMA EXPERIÊNCIA DE FORMAÇÃO PROFISSIONAL EM UMA ORGANIZAÇÃO PRIVADA}

\section{RESUMO}

Este artigo analisa as características, benefícios, desvantagens e eficácia do microlearning a partir de uma experiência de treinamento virtual fornecida a funcionários de uma seguradora privada. Os objetivos do estudo são: a) identificar as características do microlearning nos cursos de formação virtuais oferecidos aos colaboradores de uma empresa, b) descrever as vantagens e desvantagens geradas pelo microlearning, c) identificar os elementos significativos nas cápsulas de micro-conteúdo que permitiu o aprendizado. A pesquisa apresenta um desenho descritivo baseado em um estudo de caso e as técnicas de coleta de informações foram o survey e o focus group. Participam da investigação 133 profissionais de diversas disciplinas que exercem diferentes funções em uma seguradora privada. Os resultados mostram que o sucesso da microaprendizagem está associado à concepção de programas de formação profissional que considerem as idades, necessidades e exigências dos utilizadores, que obviamente têm requisitos de aprendizagem diferentes.

\section{PALAVRAS CHAVE}

Aprendizagem virtual, microlearning, formação profissional, aprendizagem de adultos, aprendizagem ao longo da vida.

\section{1.- INTRODUCCIÓN}

Desde las investigaciones realizadas por Cota y Rivera (2017), Abarca (2016) y Yunda (2012) se plantea que la formación profesional en las empresas constituye una alternativa imprescindible para lograr el éxito en los procesos de desarrollo organizacional. Por ello, 
la inversión actual en formación responde a diversos criterios como: a) especialización en la actividad laboral o disciplina, b) formación en nuevas disciplinas y tecnologías, c) actualización de los conocimientos adquiridos y d) fortalecimiento de las habilidades blandas (Gómez \& Alemán, 2011), los cuales generan nuevas perspectivas para los procesos de formación profesional (Schlemmer et al., 2007).

En este marco, el e-learning abre nuevas posibilidades de formación y desarrollo para los colaboradores en las organizaciones (Yunda, 2012), involucrando a los diversos estamentos de la organización y cambiando la cultura laboral para priorizar el potencial humano (Abarca, 2016). Estudios realizados en diversas disciplinas coinciden en señalar que el e-learning en la formación de los profesionales permite una mejor adquisición de habilidades en la autogestión, optimiza la interactividad, reflexión y práctica y, utiliza una amplia variedad de diseños y formatos de instrucción para una formación sincrónica y asincrónica (Lawn et al., 2017).

En este contexto de experiencias, el microlearning o microaprendizaje (Navarro \& Di Bernardo, 2016) surge como una modalidad o tendencia emergente del e-learning, que facilita la adquisición de competencias en tiempos donde hay una mayor movilidad y ubicuidad $y$ donde se hace necesario obtener la información en el menor tiempo posible (Trabaldo et al., 2017; Friesen, 2007), reducir costos, facilitar el acceso a cursos, generar mayores posibilidades para compartir materiales de aprendizaje, brindar flexibilidad en la planificación de horarios de estudio y reflejar la realidad emergente (Martínez, 2009; Yunda, 2012; Friesen, 2007). Por lo tanto, es una fuente de oportunidades de aprendizaje asociada a la creciente necesidad de desarrollo personal y profesional (Emerson \& Berge, 2018).

\subsection{Microlearning: un nuevo escenario de aprendizaje}

El microlearning es una modalidad de aprendizaje que permite la adquisición de conocimientos y habilidades a partir de microcontenidos, sin saturar de información al usuario (Lindner, 2006; Palazón, 2015). Se presentan actividades cortas que están interconectadas y constituidas por pequeñas unidades de información digital (Trabaldo et al., 2017) y aborda temas específicos o microcontenidos que permiten desarrollar acciones e interacciones típicas de los procesos educativos, a veces sin la necesidad de coincidir en el espacio ni en el tiempo (Mosel, 2005; Hugh, 2005; Palazón, 2015; Diaz, et al., 2020). En esta modalidad, los profesionales deciden qué aprender y en qué momento hacerlo ante un entorno laboral dinámico que exige el desarrollo de múltiples tareas en paralelo (Buchem \& Hamelmann, 2010). Los diversos dispositivos digitales (laptop, celulares inteligentes, computadoras, tabletas, etc.) con conexión a la red hacen posible habitar varios lugares al mismo tiempo y generar nuevos escenarios de aprendizaje de forma simultánea (Riveros, 2019).

El microlearning comprende: la selección del contenido, el manejo de los tiempos, el currículo, el tipo de aprendizaje, el diseño de las actividades, la mediación y la forma de presentación de los contenidos (Jomah et al., 2016; Hug, 2005; Lindner, 2006). Igualmente, en la literatura, hay estudios desarrollados en educación superior que evidencian que el uso del microlearning permite el logro de aprendizajes, facilita la evaluación formativa (Navarro \& Di Bernardo, 2016) y, constituye una estrategia didáctica útil en el aprendizaje (De Juan et al., 2012).

Por tal motivo, el presente artículo permite un análisis de las oportunidades de formación online para colaboradores de diferentes disciplinas (Lawn et al., 2017) y explora nuevas 
formas de responder a la creciente necesidad de formación que se presenta en las empresas (Salinas \& Marín, 2015).

\subsubsection{Características del microlearning}

La capacidad y la velocidad de aprender se convierte en un eje esencial del microlearning para plantear nuevos escenarios de aprendizaje (Aitchanov et al., 2013). Su presentación en contenidos específicos es una característica esencial; los cuáles son autónomos, direccionables, flexibles y digitales (Friesen, 2007) y evitan el riesgo de abrumar con un exceso de datos a los usuarios pues abordan temas o problemas simples.

Aitchanov et al. (2013) señala que, el uso de los dispositivos móviles, las portátiles y los equipos de escritorio brindan un soporte tecnológico que nos permiten estar conectados y acceder a los contenidos en cualquier momento o lugar (ubicuo). Lo planteado, configura una dinámica particular donde la brevedad constituye el valor agregado para los aprendizajes pues brinda mayor facilidad para asimilar y recordar (Trabaldo et al., 2017). De la misma manera, la usabilidad permite a los usuarios optimizar la calidad de la experiencia al interactuar con los contenidos brindados de manera sencilla (Aitchanov et al., 2013) ya sea en espacios formales o informales, diseñando contenidos digitales pertinentes a las necesidades de formación (Trabaldo et al., 2017; Aitchanov et al., 2013)

Además, es necesario que las unidades de información se elaboren en formatos pequeños que puedan ser fácilmente observados en la pantalla de los diversos dispositivos sin la necesidad de desplazarse a otras secciones. Por ello, la interfaz gráfica cumple un rol importante en el éxito del microlearning, pues brinda el entorno digital para presentar los microcontenidos; siendo necesario cuidar la calidad de los formatos digitales que presentan contenidos (Buchem \& Hamelmann, 2010).
Ahora bien, como cualquier modalidad de aprendizaje, el microlearning presenta beneficios e inconvenientes. A continuación, trataremos estos aspectos.

\subsubsection{Beneficios e inconvenientes del microlearning}

La breve cantidad de información de los microcontenidos, al ser seleccionados $y$ secuencialmente presentados a los usuarios, favorece la retención del conocimiento (Trabaldo et al., 2017) generando un gran beneficio en el proceso de formación.

Xue et al., (2010), Pyc y Rawson (2010), así como De Juan et al., (2012) coinciden en señalar que la repetición de los contenidos mejora la memoria y la capacidad de retención del conocimiento, en comparación con los cursos de formación tradicional. Por ello, es indispensable que los contenidos se ajusten al intervalo de atención que los trabajadores manifiestan. De esta manera, aumentará la motivación y los participantes del programa serán capaces de completar las distintas fases de formación (Trabaldo et al., 2017; Emerson \& Berge, 2018; Salinas \& Marín, 2015). Así también, el microlearning brinda un beneficio social pues permite reducir las brechas de conocimientos o habilidades ausentes de los colaboradores inmersos en programas de formación (Trabaldo et al., 2017).

Incorporar el microlearning en las empresas supone una alta rentabilidad, pues el proceso de implementación es mucho más ágil que los procesos de formación presencial. Lo señalado, contribuye a la creación de microcontenidos de alta calidad que pueden actualizarse periódicamente sin elevar los presupuestos de formación (Salinas \& Marín, 2015).

Sin embargo, también hay que tener en cuenta los inconvenientes que surgen a partir del microlearning cuando se omite o solo se 
aborda parcialmente sus elementos. Líneas arriba, se ha explicado que el aprendizaje debe ser desglosado en fragmentos muy pequeños de contenido para generar una grata experiencia de aprendizaje (Trabaldo et al., 2017); entonces, el desafío es crear contenidos de calidad, que brinden respuestas inmediatas a los requerimientos formativos, que permitan acompañar eficientemente las innovaciones realizadas en las empresas y genere fluidez en la comunicación sincrónica y asincrónica (Lindner, 2006).

Por otro lado, como parte de la implementación de los contenidos de calidad en las propuestas del microlearning es importante revisar el diseño del contenido de las evaluaciones que se programen. Es bien cierto, que se sugiere utilizar instrumentos de evaluación cortos como cuestionarios o preguntas de selección múltiple; sin embargo, esta acción podría terminar siendo una dificultad cuando las pruebas no permitan verificar el aprendizaje logrado; por lo tanto, hay que evitar el contenido irrelevante. Entonces, es fundamental que los contenidos se diseñen centrados en el perfil de los participantes del curso (Trabaldo et al., 2017). Un factor que constituye un inconveniente es la presentación de textos extensos que generan desmotivación; más aún, cuando se utiliza un vocabulario complejo que impide establecer un vínculo con los participantes.

Asimismo, otro aspecto que puede convertirse en un inconveniente es la funcionalidad de la plataforma. El entorno visual que presenta el micro contenido debe promover una grata experiencia para los usuarios; siendo relevante cuidar la calidad de la producción del material gráfico que se utiliza en los cursos de microlearning, pues las imágenes de baja resolución o que no corresponden con la información brindada pueden alterar la comprensión de los microcontenidos. Los videos extensos pueden terminar siendo poco motivadores (Yunda, 2012); por ello, es necesario contar con plataformas interactivas que permitan contextualizar el aprendizaje y lograr la mejora del desempeño de los colaboradores (Fugisawa \& Ferreira, 2013). Además, es indispensable considerar otros aspectos como la organización, la secuenciación de los contenidos, los recursos en la plataforma y la asistencia técnica a los participantes en todo el proceso de formación. El soporte técnico es esencial para asegurar el apoyo al usuario cada vez que lo requiera.

\subsubsection{Efectividad de las cápsulas de microcontenido}

Las cápsulas o píldoras de microcontenido generan aprendizajes inmediatos que permiten mejorar el desempeño de los colaboradores en sus actividades laborales, constituyéndose en una estrategia efectiva para programas de formación online (Salinas \& Marín, 2015; Fugisawa y Ferreira, 2013).

La efectividad, se puede producir por la gradualidad que se brinda en la experiencia formativa, pues los microcontenidos deben presentar información que se aborda desde lo más simple a lo más complejo, generando aprendizajes en escala ascendente (Trabaldo et al., 2017). De la misma manera, el tiempo de duración de la experiencia formativa en el microlearning es estratégica pues genera espacios cortos de interacción entre el usuario y el contenido lo cual permite cumplir con los objetivos de aprendizaje (Aitchanov et al., 2013; Navarro \& Di Bernardo, 2016).

Por otro lado, la repetición espaciada es otro factor que asegura la efectividad de las cápsulas pues favorece la asimilación de nuevos conceptos al ser breves (Salinas \& Marín, 2015) y permite el refuerzo de las actividades de aprendizaje. Sumado a ello, otros factores presentes son: la utilidad de los microcontenidos (los cuales deben brindarle al usuario nuevas posibilidades que permitan la mejora de su labor 
profesional), la variedad en la presentación de los microcontenidos que genera dinamicidad en la interacción del usuario con el material formativo y; finalmente, la evaluación, que como proceso verifica el logro de los aprendizajes (Aitchanov et al., 2013). En síntesis, el microlearning puede ser una opción interesante cuando las empresas deciden incluir actualizaciones o plantear nuevos aprendizajes a sus colaboradores (Emerson \& Berge, 2018).

\section{2.- METODOLOGÍA}

En la presente investigación, los objetivos del estudio han sido los siguientes: a) identificar las características del microlearning en los cursos de formación virtual brindados a los colaboradores de una empresa, b) describir los beneficios e inconvenientes que genera el microlearning, c) identificar en las cápsulas de microcontenido, los elementos significativos que permitieron el aprendizaje de los colaboradores.

La investigación se abordó desde un enfoque cualitativo pues contribuyó a comprender los significados generados en los involucrados al participar en los cursos de formación virtual (Hennink, et al., 2011 y Creswell \& Poth, 2018). Como método de investigación se seleccionó el estudio de caso de tipo descriptivo, pues se buscó interpretar una realidad particular y explicar las complejas situaciones que ocurren en su interior (Yin, 2003) describiendo la experiencia de aprendizaje virtual de los colaboradores (Gil, 2011).

A continuación, se presenta la Tabla 1 con las categorías y subcategorías de estudio.

Tabla 1

Categorías y subcategorías de estudio preliminares

\begin{tabular}{|c|c|c|c|}
\hline CATEGORÍA & \multicolumn{2}{|r|}{ SUBCATEGORÍA } & CÓDIGO \\
\hline \multirow{4}{*}{ Características del } & \multicolumn{2}{|c|}{ Accesibilidad } & $\mathrm{ACC}$ \\
\hline & \multicolumn{2}{|c|}{ Brevedad } & BRE \\
\hline & \multicolumn{2}{|c|}{ Contenido específico } & $\mathrm{CON}$ \\
\hline & \multicolumn{2}{|c|}{ Flexibilidad } & FLE \\
\hline \multirow[t]{3}{*}{ microlearning } & \multicolumn{2}{|c|}{ Personalización } & PER \\
\hline & \multicolumn{2}{|c|}{ Interfaz gráfica } & INT \\
\hline & \multicolumn{2}{|c|}{ Usabilidad } & USA \\
\hline \multirow{7}{*}{$\begin{array}{l}\text { Beneficios e inconvenientes con el } \\
\text { microlearning }\end{array}$} & \multirow{5}{*}{ BEN } & Motivación & MOT \\
\hline & & Rentabilidad & REN \\
\hline & & $\begin{array}{l}\text { Reducción de las brechas de } \\
\text { conocimiento }\end{array}$ & $\mathrm{RBC}$ \\
\hline & & Retención de conocimiento & $\mathrm{RCO}$ \\
\hline & & Soporte técnico & SOP \\
\hline & \multirow[t]{2}{*}{ INC } & Funcionalidad de la plataforma & PLA \\
\hline & & Calidad de contenidos de la evaluación & $\mathrm{CCO}$ \\
\hline \multirow{5}{*}{$\begin{array}{l}\text { Efectividad de las cápsulas de } \\
\text { microcontenido en el aprendizaje }\end{array}$} & \multicolumn{2}{|c|}{ Repetición espaciada } & REP \\
\hline & \multicolumn{2}{|c|}{ Tiempo de duración de la experiencia } & TEX \\
\hline & \multicolumn{2}{|c|}{ Variedad } & VAR \\
\hline & \multicolumn{2}{|c|}{ Utilidad } & UTI \\
\hline & \multicolumn{2}{|c|}{ Evaluación } & EVA \\
\hline
\end{tabular}

Fuente: Tomado de Hug, 2010; Aitchanov, et al., 2013; Salinas y Marín, 2014; Navarro y Di Bernardo, 2016; Trabaldo et al., 2017; Emerson and Berge, 2018. 


\subsection{Participantes}

Los participantes de la investigación fueron 133 profesionales de diversas disciplinas que laboran en diversos puestos de trabajo en una empresa privada de seguros. Del total de participantes, el
$68 \%$ eran mujeres y el $32 \%$ fueron hombres, de los cuales el $14.3 \%$ tenía entre 20 a 30 años, el $26.3 \%$ entre 31 a 40 años, el $33.8 \%$ entre 41 a 50 años, $21.8 \%$ entre 51 a 60 años y $3.8 \%$ tenía más de 61 años. La descripción detallada de los colaboradores se puede observar en la Tabla 2.

Tabla 2

Participantes de las diversas áreas de trabajo de la empresa

\begin{tabular}{|c|c|c|c|c|c|}
\hline Área de trabajo & $\begin{array}{c}\mathrm{N}^{\circ} \text { de } \\
\text { participantes }\end{array}$ & Porcentaje & Femenino & Masculino & $\begin{array}{c}\text { Media de edad de } \\
\text { participantes }\end{array}$ \\
\hline Actuarial & 4 & $3.0 \%$ & 1 & 3 & 29 \\
\hline $\begin{array}{l}\text { Administración } \\
\text { y Tesorería }\end{array}$ & 3 & $2.3 \%$ & 2 & 1 & 44 \\
\hline $\begin{array}{l}\text { Analítica y } \\
\text { Riesgos Técnicos }\end{array}$ & 3 & $2.3 \%$ & 0 & 3 & 37 \\
\hline $\begin{array}{l}\text { Comercial } \\
\text { Masivos }\end{array}$ & 5 & $3.8 \%$ & 3 & 2 & 34 \\
\hline Contabilidad & 6 & $4.5 \%$ & 4 & 2 & 38 \\
\hline $\begin{array}{l}\text { Cultura } \\
\text { Organizacional }\end{array}$ & 2 & $1.5 \%$ & 1 & 1 & 26 \\
\hline Gerencia General & 1 & $0.8 \%$ & 1 & 0 & 45 \\
\hline Inversiones & 3 & $2.3 \%$ & 1 & 2 & 45 \\
\hline $\begin{array}{l}\text { Legal y } \\
\text { Compliance }\end{array}$ & 2 & $1.5 \%$ & 1 & 1 & 32 \\
\hline $\begin{array}{l}\text { Marketing y } \\
\text { Experiencia de } \\
\text { clientes }\end{array}$ & 2 & $1.5 \%$ & 2 & 0 & 27 \\
\hline $\begin{array}{l}\text { Operaciones y } \\
\text { Tecnología }\end{array}$ & 16 & $12.0 \%$ & 10 & 6 & 35 \\
\hline Rentas Vitalicias & 85 & $63.9 \%$ & 64 & 21 & 47 \\
\hline $\begin{array}{l}\text { Soluciones de } \\
\text { clientes }\end{array}$ & 1 & $0.8 \%$ & 1 & 0 & 45 \\
\hline Total & 133 & $100 \%$ & 91 & 42 & \\
\hline
\end{tabular}

Fuente: elaboración propia. 
Estos 133 profesionales participaron en 41 cursos de formación (desarrollados de forma paralela en el transcurso de nueves meses), entre los cuales se brindaron cursos obligatorios y electivos, dependiendo del área de trabajo y las necesidades del puesto.

\subsection{Instrumentos}

Para el recojo de información se aplicó una encuesta y un grupo focal (Fàbregues et al., 2016; Arboleda, 2008). La encuesta fue virtual y participaron 133 colaboradores de los diferentes cursos del programa de formación. Se elaboró para ello un cuestionario con 17 ítems con la finalidad de recoger información sobre las características del microlearning, los beneficios que brinda y la efectividad de los microcontenidos. El grupo focal se llevó a cabo con un grupo de 8 colaboradores que fueron seleccionados de las diversas áreas de trabajo. Se realizaron preguntas referidas al desarrollo de los cursos de formación y luego, se transcribieron las respuestas asignando a las intervenciones de los participantes, un código con el siguiente formato [C1, MOT, p.1], donde se aprecian tres elementos descriptores: (1) el número de identificación del colaborador; (2) la abreviatura de la subcategoría que se identifica en la intervención y (3) la página de la transcripción donde se encuentra la cita.
Para el tratamiento de los datos recogidos en la encuesta se utilizó el paquete estadístico SPSS versión 22.0, a partir del cual se realizó un análisis descriptivo. En el caso, del grupo focal se utilizó el análisis de contenido como técnica para realizar el análisis de las transcripciones (Gibbs, 2012). Con el fin de asegurar la confidencialidad, los nombres de todos los colaboradores se mantuvieron en reserva y fueron codificados para el análisis. Es importante destacar que cada colaborador recibió el protocolo de consentimiento informado.

\section{3.- ANÁLISIS Y RESULTADOS}

A continuación, se muestran los resultados obtenidos en cada categoría:

\section{a. Características del microlearning}

Concluido los cursos de formación virtual en modalidad microlearning, se solicitó a los colaboradores resolver una encuesta. Los resultados obtenidos (Tabla 3) ponen de manifiesto que los colaboradores identifican mayoritariamente como características de los cursos: la brevedad (3.53), la accesibilidad (3.37), la usabilidad (3.52), la personalización (3.28) y la interfaz gráfica (3.52). 
Tabla 3

Estadísticos descriptivos de la percepción de los participantes sobre las características de los cursos de microlearning

\begin{tabular}{|l|c|c|c|c|c|c|}
\hline \multicolumn{1}{|c|}{ Ítems } & \multirow{2}{*}{$\mathrm{N}^{\circ}$} & TD* & $\mathrm{D}$ & $\mathrm{A}$ & $\mathrm{TA}$ & Media \\
\begin{tabular}{|l|c|c|c|} 
Me agradó que los cursos que se \\
brindaron fueran cortos.
\end{tabular} & 113 & $2 \%$ & $2 \%$ & $36 \%$ & $59 \%$ & 3.53 \\
\hline $\begin{array}{l}\text { El curso funcionó en distintos } \\
\text { dispositivos de comunicación (PC, } \\
\text { tabletas, móviles). }\end{array}$ & 113 & $2 \%$ & $2 \%$ & $36 \%$ & $59 \%$ & 3.37 \\
\hline $\begin{array}{l}\text { Las acciones que se realizaron en la } \\
\text { plataforma del curso fueron fáciles de } \\
\text { ejecutar. }\end{array}$ & 113 & $2 \%$ & $2 \%$ & $36 \%$ & $59 \%$ & 3.52 \\
\hline $\begin{array}{l}\text { La organización del curso se desarrolló } \\
\text { teniendo en cuenta las características } \\
\text { de los participantes del curso. }\end{array}$ & 113 & $2 \%$ & $2 \%$ & $36 \%$ & $59 \%$ & 3.28 \\
\hline $\begin{array}{l}\text { El curso brindó mensajes o alertas de } \\
\text { forma oportuna que notificaron sobre la } \\
\text { importancia de revisar los contenidos del } \\
\text { mismo. }\end{array}$ & 113 & $2 \%$ & $2 \%$ & $36 \%$ & $59 \%$ & 3.39 \\
\hline $\begin{array}{l}\text { Las imágenes y objetos gráficos de } \\
\text { la plataforma presentaron un entorno } \\
\text { visual sencillo que permitió su uso. }\end{array}$ & 113 & $2 \%$ & $2 \%$ & $36 \%$ & $59 \%$ & 3.52 \\
\hline
\end{tabular}

Nota: $\left(^{*}\right)$ TD Totalmente en desacuerdo, D En desacuerdo, A De acuerdo, TA Totalmente de acuerdo

Además, los colaboradores en el grupo focal coincidieron en afirmar que la flexibilidad fue una característica esencial en los cursos, pues brindó la posibilidad de organizar sus propios horarios de estudio e incluso, se pudo aprovechar los momentos usados para el desplazamiento a sus hogares y la hora de almuerzo.

Bueno normalmente yo no le dedico 100 por ciento cuando empiezo algo, o sea voy entrando por momentos. [C1, FLE, p.11]

Yo intento hacerlo en mi hora de almuerzo porque de ahí es bien complicado. [C4, FLE, p.16]

Yo vivo lejos y me demoro bastante en llegar a mi casa y a mí me gusta bastante en ese caso leer en el carro y yo tenía planeado llevar esos cursos aprovechando ese tiempo. [C5, ACC, p.29]

En opinión de los colaboradores, la interfaz gráfica de los cursos en la plataforma presentó un entorno visual muy sencillo que permitió su uso sin ningún inconveniente.

A mí me ha parecido en realidad súper interactivo porque tiene un poco de todo, tiene una parte de video, luego tiene un quiz simple.... [C2, INT, p.4]

Había bastantes videos y ese tipo de lectura era mucho más rápido o imágenes que te hacían como que fuera un poco más entretenido y no te costará tanto leerlo. [C6, INT, p.11] 
A diferencia de otros cursos virtuales que alguna vez he llevado, me parece más amigable, más entretenido, no tan aburrido. [C7, INT, p.5]

Como parte de las bondades de la interfaz gráfica, los colaboradores recibieron notificaciones de forma progresiva, las cuales fueron bien apreciadas pues permitió que organicen su tiempo.

Te llega un mensaje al inicio del trimestre y te dice "ya están habilitados los cursos", luego en quincena, [...] la última semana antes que cierre te avisan. [C3, INT, p.16]

Asimismo, los colaboradores reconocen que el diseño de los cursos estuvo centrado en sus intereses y necesidades de formación.

Hay una malla curricular de otras áreas [se refiere a otras mallas de cursos] que simplemente son pensadas en el perfil de la persona y si ellos tienen tiempo $y$ quieren hacerlo pueden realizarlo. [C4, $P E R$ y $R B C, p .17]$

Los cursos que tenemos están contemplados según el perfil de la persona. [C7, PER, p.24]
Por otro lado, también valoraron que los cursos hayan sido cortos pues, la brevedad de los mismos, permitió abordar contenidos específicos que reforzaron sus conocimientos.

Para el día a día te ayuda que sea resumido. [C7, BRE, p.12]

Me pareció que si eran resumidos los cursos y eso era bueno [...] nosotros por trimestre tenemos una cantidad de cursos que son entre 3 a 4 cursos por trimestre $y$ de hecho llevar de 3 a 4 cursos con la evaluación es el doble. [C3, BRE, p.3]

Los colaboradores coincidieron en afirmar que desarrollaron los cursos en la plataforma sin inconvenientes; asimismo, reconocieron como un beneficio la accesibilidad en diversos dispositivos.

La plataforma me pareció fácil de utilizar, es súper intuitiva. [C3, USA, p.3]

Cuando desarrollé los cursos no he tenido ningún inconveniente. [C2, USA, p.11]

No sé si a ustedes les ha pasado, pero por ejemplo yo primero descargué la aplicación en mi teléfono... [C6, ACC, p.8]

En los fragmentos del discurso de los colaboradores se identificaron las características del microlearning, tal como se detalla en la Figura 1. 


\section{Figura 1}

Análisis sobre las características del microlearning.

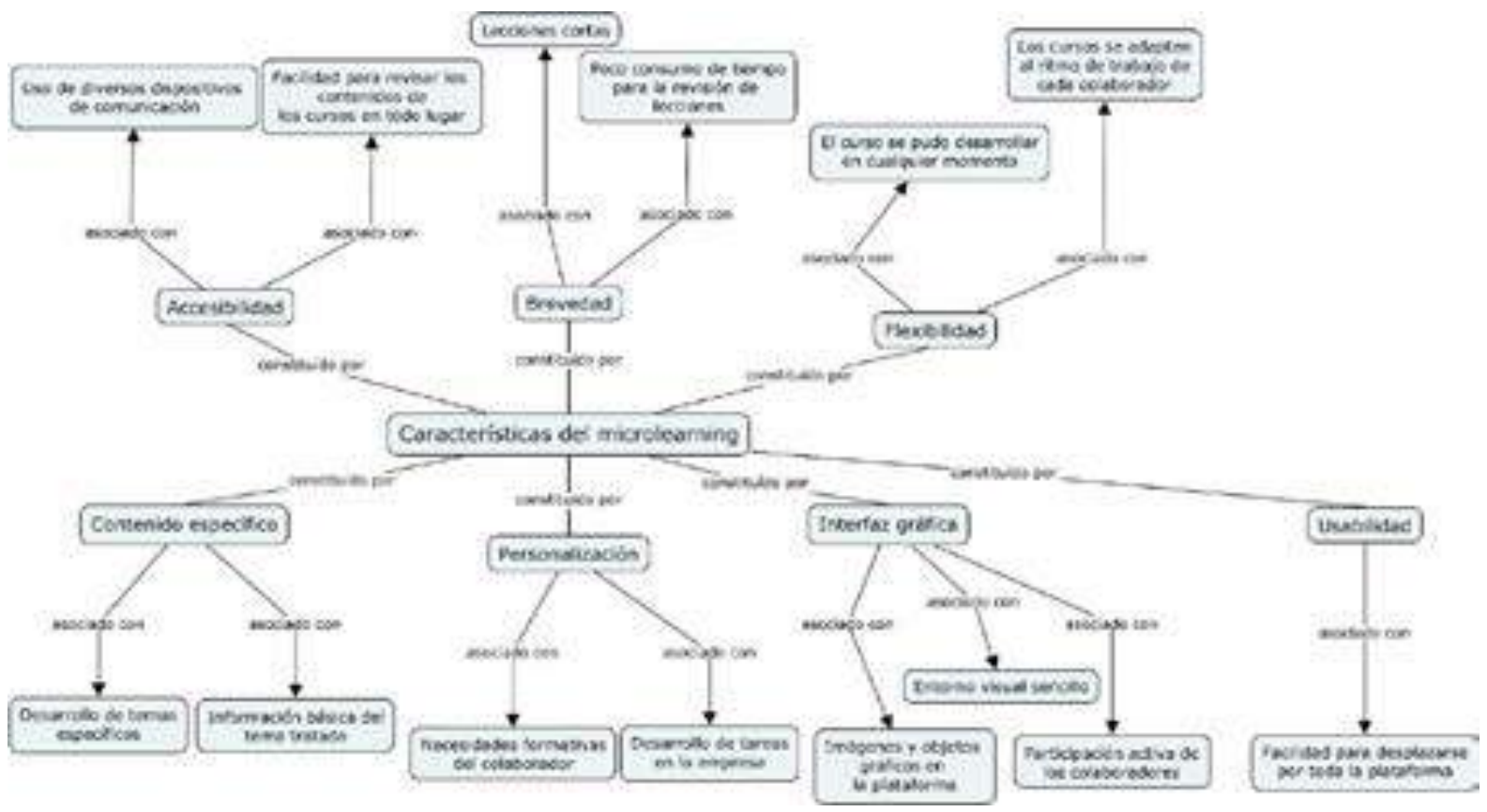

Fuente: elaboración propia.

\section{b. Beneficios e inconvenientes con el} microlearning

Los colaboradores pusieron de manifiesto que la motivación constituyó un componente relevante al incorporar el microlearning en el proceso de formación. Asimismo, reconocieron que la organización de los cursos permitió optimizar la rentabilidad en contraste a una formación presencial. En conjunto, el microlearning posibilitó reducir las brechas del conocimiento, pues los colaboradores tuvieron acceso a cursos electivos en razón de su disponibilidad de tiempo y deseos de aprender (Tabla 4).

Tabla 4

Estadísticos descriptivos de la percepción de los participantes sobre los beneficios que brinda el microlearning

\begin{tabular}{|l|c|c|c|c|c|c|}
\hline \multicolumn{1}{|c|}{ İtems } & $\mathrm{N}^{*}$ & \multicolumn{2}{|c|}{$\%$} & Media \\
\hline $\begin{array}{l}\text { La distribución del texto e imágenes } \\
\text { resultaron muy motivadores e interesantes. }\end{array}$ & 113 & $2 \%$ & $5 \%$ & $44 \%$ & $50 \%$ & 3.41 \\
\hline $\begin{array}{l}\text { Me gustó que el desarrollo del curso sea } \\
\text { virtual. }\end{array}$ & 113 & $2 \%$ & $5 \%$ & $32 \%$ & $62 \%$ & 3.55 \\
\hline $\begin{array}{l}\text { La información que se presentó en los } \\
\text { contenidos del curso fue fácil de entender. }\end{array}$ & 113 & $1 \%$ & $4 \%$ & $37 \%$ & $59 \%$ & 3.53 \\
\hline $\begin{array}{l}\text { Los contenidos respondieron a la realidad } \\
\text { laboral y profesional. }\end{array}$ & 113 & $4 \%$ & $6 \%$ & $39 \%$ & $51 \%$ & 3.38 \\
\hline
\end{tabular}

Nota: $\left({ }^{*}\right)$ TD Totalmente en desacuerdo, D En desacuerdo, A De acuerdo, TA Totalmente de acuerdo 
En el grupo focal, los colaboradores coincidieron en reconocer y valorar que la empresa de seguros les brindó un programa de formación virtual con cursos obligatorios, afines a sus áreas de trabajo, y también, cursos electivos (no obligatorios); que en conjunto les brindó la oportunidad de ampliar los conocimientos y aprendizajes de todos los colaboradores.

Hay como más de 50 cursos, en el que puedes escoger como redacción, plan de marketing, KPl's, Balance Score Card...hay varios, que son de hecho la parte obligatoria en el caso de jefes que tienen un tipo de malla, los analistas también tienen otro tipo de malla y los asistentes tienen otro tipo de malla, que están enfocados más que todo en sus habilidades y lo que se quiere potenciar en ese momento de su posición. [C6, RBC, p.25]

Estado Financiero no lo vemos porque no estoy en área de finanzas, pero como que es bueno saberlo y aparte nosotros de comercial siempre cuando vas hacia el cliente tienes que saber algún tipo de indicador ¿no? No solo de tu compañía sino de tu área. [C1, RBC, p.23]
Ofrecer un programa de formación en modalidad microlearning con un abanico diverso de cursos permitió reducir las brechas del conocimiento. La malla curricular abordó cursos específicos que se desarrollaron en plazos breves y demandó poco presupuesto optimizando la rentabilidad; asimismo, los contenidos breves posibilitaron la rápida asimilación de los conocimientos, fortaleciendo la motivación en sus colaboradores.

Más allá de que sí es básico o no, sea habilidades blandas o no, es algo que nos está dando la empresa extra y que normalmente el año pasado no teníamos, no teníamos capacitación corporativa y no teníamos nada de eso. [C4, REN y MOT, p.24]

En el día a día no tienes todos los conocimientos claros en la cabeza, siempre hay un término o algo que ya te olvidaste y lo recuerdas y si en algún momento necesitas aplicarlo o estas en una conversación y están hablando de un tema parecido como que puedes seguir continuando con el diálogo ... [C2, RCO, p.24]

En la Figura 2 se presentan las voces de los colaboradores, en cuanto a los beneficios e inconvenientes que identificaron con el microlearning. 


\section{Figura 2}

Análisis sobre los beneficios del microlearning.

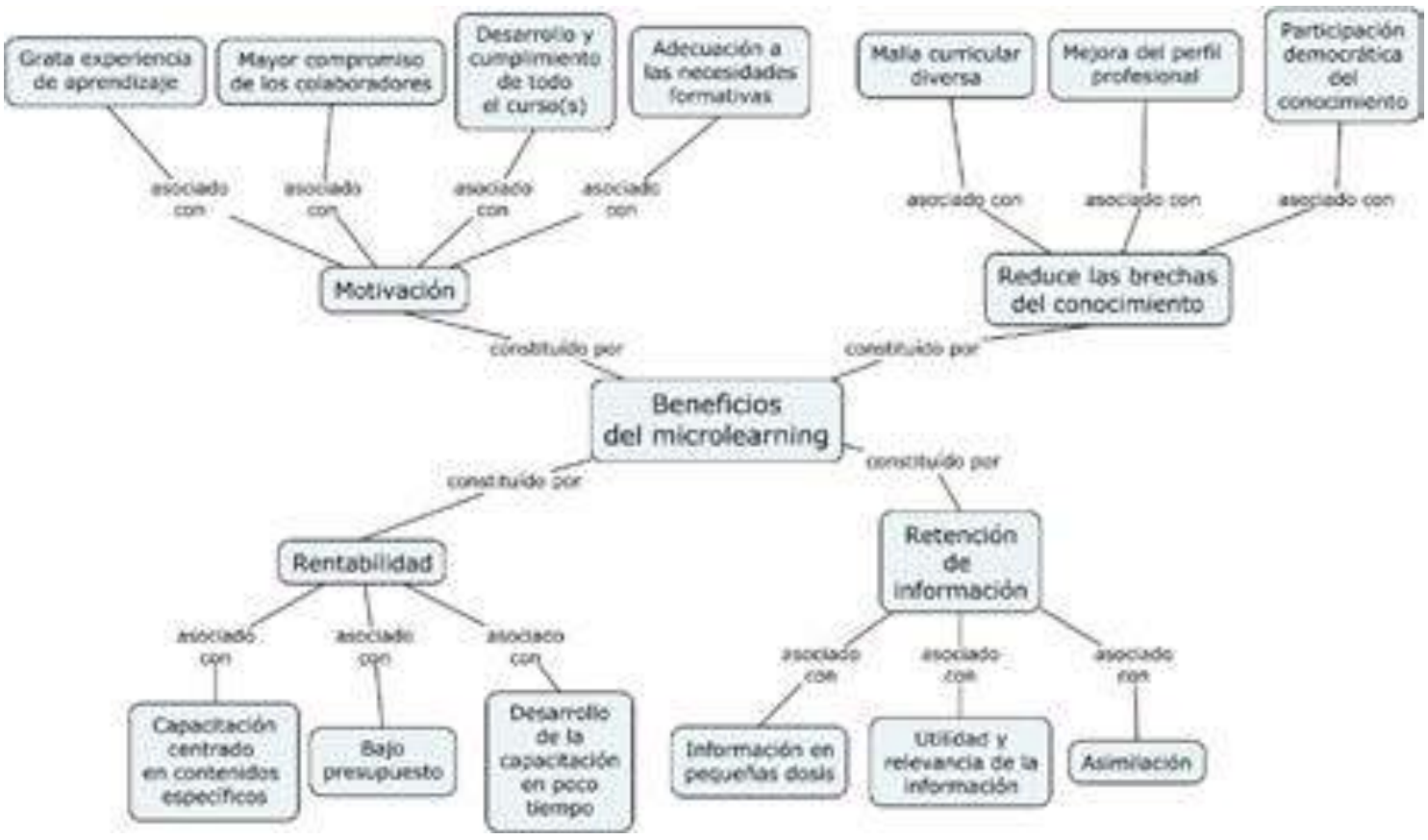

Fuente: elaboración propia.

90 Por el contrario, algunos colaboradores manifestaron que durante el desarrollo de los cursos se presentaron algunos inconvenientes entre las cuales destacaron: a) funcionalidad de la plataforma donde desarrollaron los cursos pues hubo enlaces que no cargaron, b) contenidos de la evaluación, los cuales fueron poco relevantes en opinión de los colaboradores y c) soporte técnico pues no se contó con la asistencia inmediata cuando surgieron dificultades con la plataforma.

Los links no funcionaban, no cargaban... [C1, PLA, p.10]

Es demasiado textual la respuesta [de la evaluación] no te permite razonar un poco más o analizar lo que has estado leyendo previamente. [C6, EVA, p.10]
Sería bueno que la plataforma te brinde preguntas aleatorias para evitar cosas que podrían pasar. [C3, EVA, p.31]

En la computadora sale, cuando tienes problemas ingresa aquí y también te decía comunícate con el soporte de tu empresa. [C7, SOP, p.10]

En resumen, los inconvenientes que se identificaron con el microlearning fueron los siguientes (Figura 3): 


\section{Figura 3}

Análisis sobre los inconvenientes del microlearning.

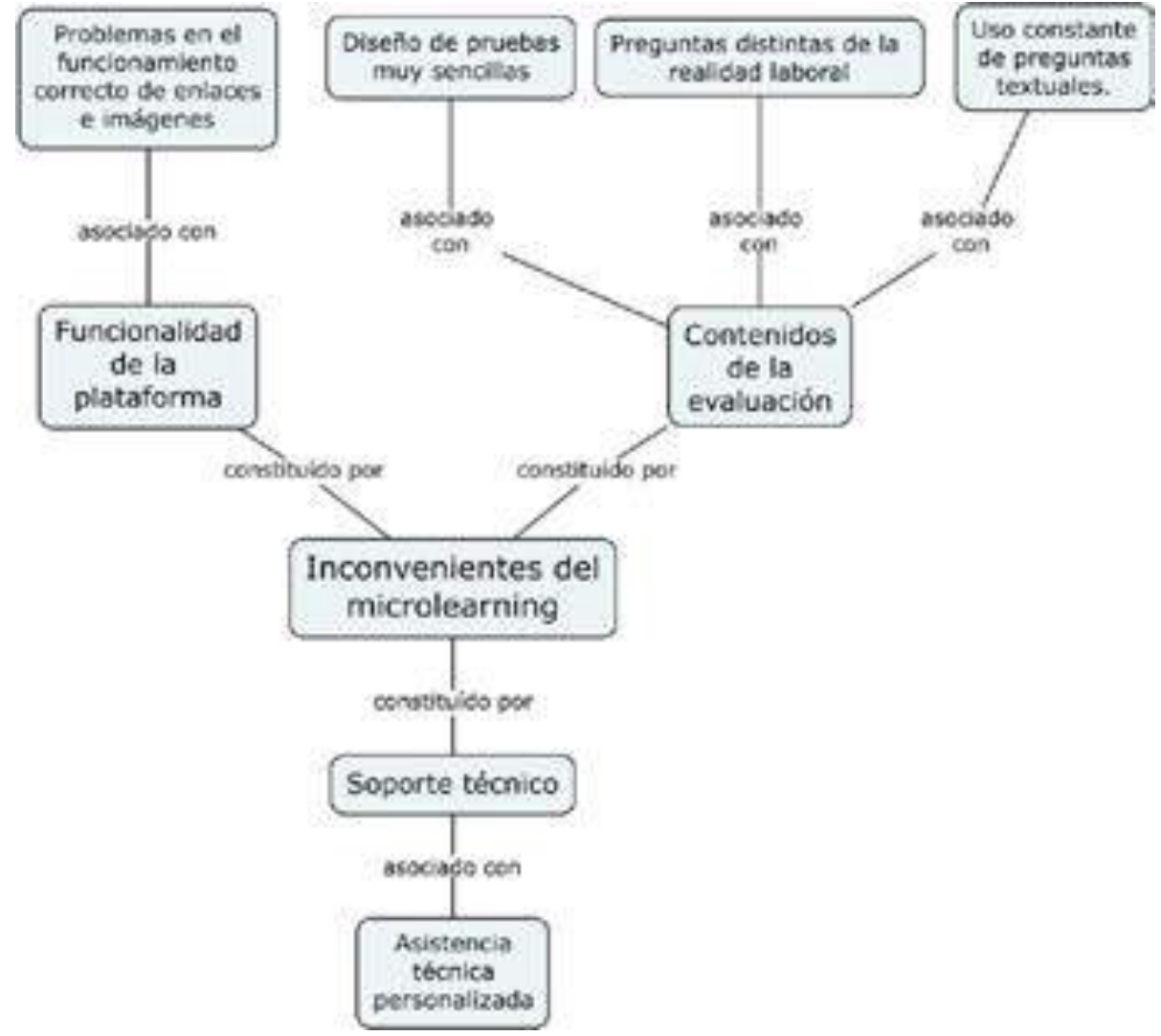

Fuente: elaboración propia.

c. Efectividad de las cápsulas de microcontenido en el aprendizaje

Los colaboradores manifestaron que la utilidad y la variedad constituyeron factores decisivos que intervinieron en el logro de la efectividad de las cápsulas de microcontenido. De la misma manera, apreciaron que la repetición espaciada logró reforzar periódicamente los aprendizajes y; además, reconocieron que la evaluación fue un factor indispensable para determinar la efectividad de las cápsulas de microcontenido en la mejora de la actividad laboral (Tabla 5). 
Tabla 5

Estadísticos descriptivos de la percepción de los participantes sobre la efectividad de las cápsulas de microcontenido en el aprendizaje.

\begin{tabular}{|c|c|c|c|c|c|c|}
\hline \multirow{2}{*}{ Ítems } & \multirow{2}{*}{$\mathrm{N}^{\circ}$} & \multicolumn{4}{|c|}{$\%$} & \multirow{2}{*}{ Media } \\
\hline & & $\mathrm{TD}^{*}$ & $D^{*}$ & $A^{*}$ & $\mathrm{TA}^{*}$ & \\
\hline $\begin{array}{l}\text { Cada tema desarrollado presentó videos, juegos, } \\
\text { diapositivas o preguntas de discusión. }\end{array}$ & 113 & $2 \%$ & $4 \%$ & $46 \%$ & $48 \%$ & 3.40 \\
\hline $\begin{array}{l}\text { El curso brindó suficiente información que } \\
\text { fortaleció mis conocimientos sobre el trabajo. }\end{array}$ & 113 & $3 \%$ & $7 \%$ & $36 \%$ & $54 \%$ & 3.41 \\
\hline $\begin{array}{l}\text { Para la mejor comprensión de los contenidos se } \\
\text { presentaron esquemas, figuras o tablas. }\end{array}$ & 113 & $2 \%$ & $3 \%$ & $46 \%$ & $49 \%$ & 3.41 \\
\hline $\begin{array}{l}\text { Repetir los temas cada cierto tiempo me permitió } \\
\text { recordar lo aprendido. }\end{array}$ & 113 & $1 \%$ & $8 \%$ & $41 \%$ & $50 \%$ & 3.40 \\
\hline $\begin{array}{l}\text { Cada tema presentó casos prácticos que me } \\
\text { permitieron comprender los contenidos. }\end{array}$ & 113 & $2 \%$ & $8 \%$ & $42 \%$ & $49 \%$ & 3.38 \\
\hline $\begin{array}{l}\text { Las preguntas tipo test que se presentaron al } \\
\text { final del curso me permitieron evaluar todo lo } \\
\text { aprendido. }\end{array}$ & 113 & $2 \%$ & $6 \%$ & $41 \%$ & $50 \%$ & 3.40 \\
\hline $\begin{array}{l}\text { Pude revisar los contenidos del curso tantas } \\
\text { veces como lo requería. }\end{array}$ & 113 & $1 \%$ & $9 \%$ & $43 \%$ & $47 \%$ & 3.37 \\
\hline
\end{tabular}

Nota: (*) TD Totalmente en desacuerdo, D En desacuerdo, A De acuerdo, TA Totalmente de acuerdo

En el grupo focal, los colaboradores reconocieron diversos factores que permitieron lograr que las cápsulas de aprendizaje resulten efectivas. Entre los factores mencionados, hubo coincidencias al señalar que la utilidad era altamente importante en el aprendizaje, pues los colaboradores necesitan que, los microcontenidos estén orientados a usos diversos de su actividad profesional.

Es súper didáctico, [...] te ayuda efectivamente en tu día a día. [C2, UTI, p.12]

Si, por más que sean básicos para muchas personas de repente los conocemos o no, pero si es como un recordaris. [C2, UTI, p.23]

De la misma manera, la repetición espaciada se constituyó en otro factor significativo para lograr mayor efectividad en los microcontenidos.
Los colaboradores valoraron que, cada cierto periodo de tiempo, se planteara un repaso de lo previamente desarrollado.

Lo que me gusta es que puedo volver a entrar y puedo retomar donde me quede. [C7, REP, p.12]

De hecho, me ayudó a reforzar y creo que a varias personas también les pasó eso. [C6, REP, p.22]

Hay que destacar que durante las intervenciones en el grupo focal otros tres factores que nombraron los trabajadores fueron: la variedad de formatos de presentación del microcontenido (juegos, vídeos, diapositivas, preguntas, etc.), el tiempo de duración de la experiencia formativa y la evaluación, como un factor que permite comprobar el logro de los aprendizajes. 
Algunas imágenes que presentan algunos casos, te ayudan a recordar, por ejemplo, recuerdo bastante el de Balance Score Card, te pone el caso de dos pilotos, que cuando tú no tienes indicadores, el avión se puede estrellar, si bien tú no puedes recordar bien que significa ese concepto ya con el caso te da a entender más o menos de que trata. [C8, VAR, EVA p.34]
Durante tu día a día es complicado, pero como eran cortos [los cursos] eran más fáciles hacerlos. [C8, BRE, p.5]

En la Figura 4 se muestran las voces de los colaboradores de la empresa de seguros quienes identificaron los factores presentes en garantizar la efectividad de las cápsulas de microcontenido.

Figura 4

Análisis sobre la efectividad de las cápsulas de microcontenido en el aprendizaje.

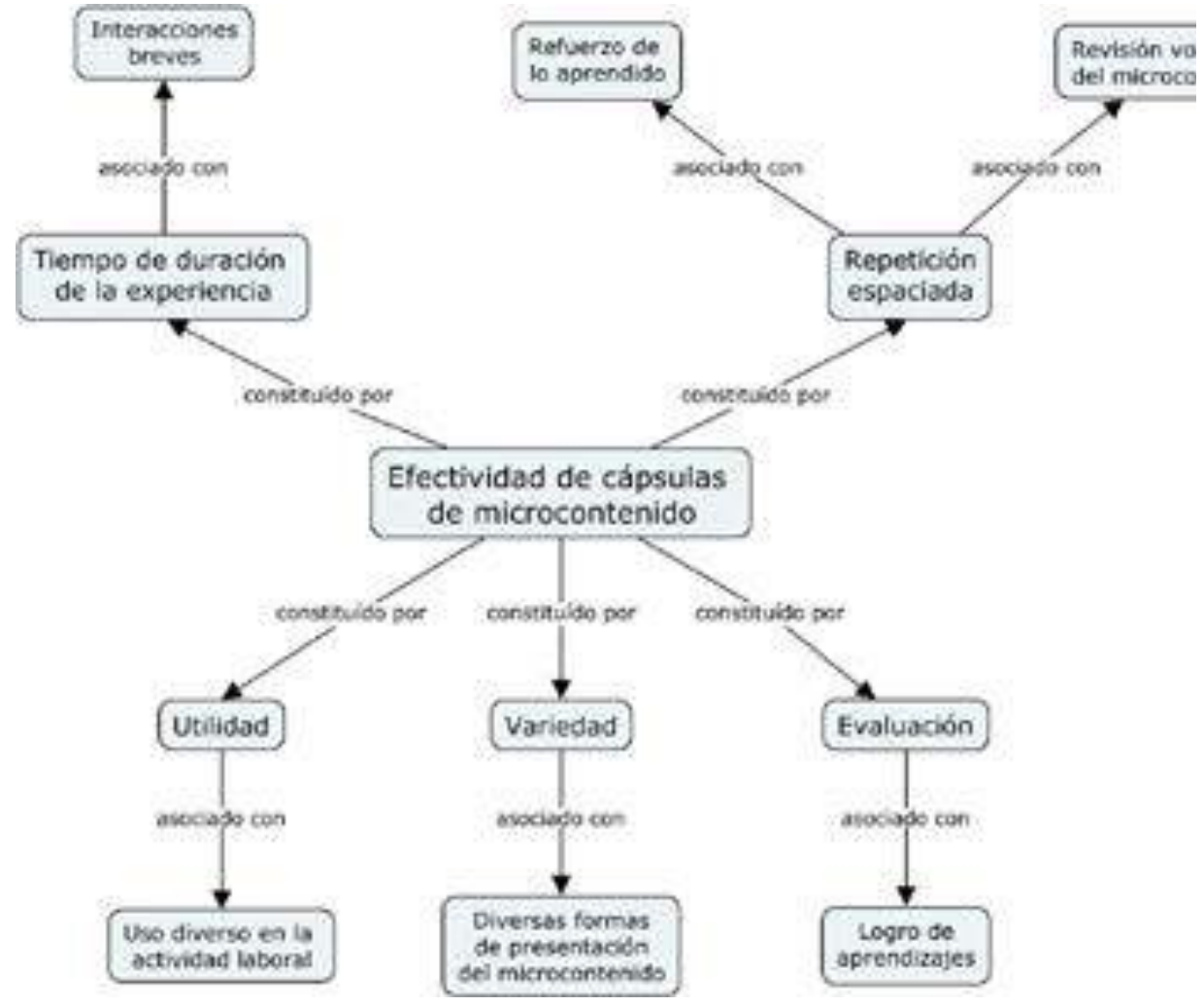

Fuente: elaboración propia.

\section{4.- DISCUSIÓN}

Este estudio ha puesto de manifiesto que la incorporación del microlearning en el proceso de formación profesional en una empresa es de vital importancia pues permite fortalecer las competencias laborales de los colaboradores. De esta manera, se constituye en una modalidad de aprendizaje "just in time" que permite atender múltiples necesidades en poco tiempo (Lindner, 2006; Trabaldo, Mendizábal \& González, 2017; Mosel, 2005) en medio de un entorno laboral dinámico y cambiante que exige a los colaboradores el desarrollo de múltiples tareas en paralelo (Hugh, 2005; Buchem \& Hamelmann, 2010). 
Como resultado de la experiencia vivida, los colaboradores del estudio identificaron en el microlearning las siguientes características: i) accesibilidad, ii) brevedad, iii) flexibilidad, iv) contenido específico, v) personalización, vi) interfaz gráfica y vii) usabilidad. Estas características halladas en el estudio responden a un entorno laboral cada vez más dinámico y digital que, como Friesen (2007) señala, exige abordar la formación profesional de forma simple y flexible, replanteando el diseño y tomando en cuenta las edades, necesidades y demandas de los usuarios, quienes evidentemente tienen requerimientos de aprendizaje diferente (Trabaldo et al., 2017; Aitchanov et al., 2013). Lo expuesto evidencia que la formación en adultos debe diseñarse a "la medida" para promover aprendizajes en cualquier lugar o momento (De Juan et al., 2012, Diaz, et al., 2020) con el uso de los diversos dispositivos digitales (Riveros, 2019).

En cuanto a los beneficios del microlearning se identificaron los siguientes elementos: i) motivación, ii) rentabilidad, iii) retención de la información y iv) reducción de las brechas del conocimiento. Es importante destacar estos beneficios pues, de acuerdo con Salinas y Marín (2015), permite elevar los niveles de compromiso, participación e interacción de los colaboradores en la formación virtual. De la misma manera, resulta rentable, pues termina siendo útil para todos los colaboradores de la empresa, permitiendo así, una participación democrática del conocimiento (Trabaldo et al., 2017; Lindner, 2006). Es así que, el microlearning constituye una alternativa para aquellas empresas que se proponen promover aprendizajes entre sus colaboradores de una manera eficiente; optimizando el uso del tiempo dedicado al aprendizaje (Navarro \& Di Bernardo, 2016).

El estudio también evidencia los inconvenientes que se generan en el desarrollo del microlearning cuando no se planifica de forma asertiva el proceso de formación profesional. Los inconvenientes identificados fueron: i) problemas en la funcionalidad de la plataforma, ii) contenidos de evaluación distantes de la realidad del colaborador y iii) falta de asistencia técnica personalizada a los colaboradores que participaron de los cursos de formación. Es importante tener presente que los adultos buscan encontrar en la plataforma un entorno visual con material gráfico de calidad que les permita revisar de manera sencilla contenidos prácticos y útiles relacionados con la actividad que realizan (Trabaldo et al., 2017; Yunda, 2012; Fugisawa \& Ferreira, 2013).

Finalmente, con respecto a la efectividad de las cápsulas de microcontenido, se identificaron los siguientes elementos: i) utilidad, ii) variedad, iii) evaluación, iv) repetición espaciada y v) tiempo de duración de la experiencia. Como se puede apreciar, los colaboradores valoraron las interacciones breves (Aitchanov et al., 2013, Lindner, 2006; Trabaldo et al., 2017) y la repetición espaciada de los contenidos, pues permitió cumplir con el logro de los objetivos (Aitchanov et al., 2013; Emerson \& Berge, 2018).

En definitiva, el éxito del microlearning dependerá de la capacidad de elaborar propuestas formativas que tomen en consideración el ritmo de vida actual de los colaboradores y sus requerimientos formativos para garantizar aprendizajes permanentes. 


\section{REFERENCIAS BIBLIOGRÁFICAS}

Abarca, Y. (2016). Procesos de capacitación ayudados por TIC en el ámbito laboral. Revista de Lenguas Modernas, (25), 343-354. https://bit.ly/3gphP8f

Aitchanov, B. H., Satabaldiyev, A. B., \& Latuta, K. N. (2013). Application of microlearning technique and Twitter for educational purposes. Journal of Physics: Conference Series, 423(1), 1-5. https:// iopscience.iop.org/article/10.1088/1742-6596/423/1/012044/pdf

Arboleda, L. M. (2008). El grupo de discusión como aproximación metodológica en investigaciones cualitativas. Revista Facultad Nacional de Salud Pública, 26(1), 69-77. https://www.redalyc.org/ pdf/120/12026111.pdf

Buchem, I. \& Hamelmann, H. (2010). Microlearning: a strategy for ongoing professional development. Documentos de aprendizaje electrónico, 21(7), 1-15. https://bit.ly/2MOMUAZ

Cota, J. A., \& Rivera, J. L. (2017). La capacitación como herramienta efectiva para mejorar el desempeño de los empleados. Técnica administrativa, 16(70). https://dialnet.unirioja.es/servlet/ articulo?codigo $=6056989$

Creswell, J. \& Poth, C. (2018). Qualitative inquiry \& research design: choosing among five approaches. SAGE Publications.

De Juan, J., Pérez, R. M., Vizcaya, M., Romero A., Girela, J., Gomez, M.J., Segovia, Y. \& Martínez, N. (2012). Microaprendizaje, reconocimiento de patrones e interacción con el entorno: estrategias didácticas para un aprendizaje eficaz. In $X$ Jornades de Xarxes d'Investigació en Docència Universitària: la participació i el compromís de la comunitat universitària (pp. 3262-3278). Instituto de Ciencias de la Educación. http://rua.ua.es/dspace/handle/10045/24277

Diaz, J. J., Salinas, H. A., Saucedo, M. S., y Jiménez, S. J. (2020). Aproximación en el uso de EVA en estudiantes de educación superior. Revista Boletín Redipe, Vol. 9, №11, 2020, 98-109.

https://revista.redipe.org/index.php/1/article/view/1113

Emerson, L. C., \& Berge, Z. L. (2018). Microlearning: Knowledge management applications and competency-based training in the workplace. UMBC Faculty Collection. http://www.kmel-journal. org/ojs/index.php/online-publication/article/view/17/17

Fàbregues, S., Meneses, J., Rodríguez-Gómez, D. \& Paré, M. H. (2016). Técnicas de investigación social y educativa. Editorial UOC. https://cutt.ly/gyTeJ8K

Friesen, N. (2007). The Microlearning Agenda in the Age of Educational Media. In: Martin Lindner and Peter A. Bruck (eds.): Micromedia and Corporate Learning. Proceedings of the 3rd International Microlearning 2007, Innsbruck University Press, 63-78.

Fugisawa, M. I. \& Ferreira, S. (2013). Microcontenido educativo para aprendizaje con movilidad: propuesta de modelo de producción. In Embrapa Informática Agropecuária-Artigo em anais de congresso (ALICE). In: Congreso Internacional de Ciudades Creativas, 3., 2013, Campinas. Actas. Madrid: ICONO14, 2013. 
Gibbs, G. (2012). El análisis de datos en investigación cualitativa. Ediciones Morata.

Gil, R. (2011). Diseño de proyectos de investigación cualitativa. Noveduc Libros.

Gómez, M. \& Alemán, L. (2011). Administración de proyectos de capacitación basados en tecnología. https://bit.ly/2LZPZ4g

Hennink, M; Hutter, I, \& Bailey, A. (2011). Qualitative Research Methods. British Library.

Hug, T. (2005). Micro Learning and narration. Exploring possibilities of utilization of narrations and storytelling for the designing of "micro units" and didactical micro-learning arrangements. In Proceedings of the International Conference, Media in Transition 4: The Work of Stories. Cambridge, MA: MIT Press. https://bit.ly/3c6KYBA

Jomah, O., Masoud, A. K., Kishore, X. P. \& Sagaya, A. (2016). Micro learning: A modernized education system. BRAIN. Broad Research in Artificial Intelligence and Neuroscience, 7(1), 103-110. http:// www.edusoft.ro/brain/index.php/brain/article/view/582/627

Lawn, S., Zhi, X., \& Morello, A. (2017). An integrative review of e-learning in the delivery of selfmanagement support training for health professionals. BMC Medical Education, 17(183), 1-16. https://doi.org/10.1186/s12909-017-1022-0

Lindner, M. (2006). Use these tools, your mind will follow. Learning in immersive micromedia and microknowledge environments. In The next generation: Research proceedings of the 13th ALT-C conference (pp. 41-49). https://bit.ly/2B38PW7

Martínez, E. M. (2009). La gestión del conocimiento a través del e-learning. Un enfoque basado en escenarios. Investigaciones Europeas de Dirección y Economía de la Empresa, 15(1), 29-44. https://www.sciencedirect.com/science/article/pii/S1135252312600768

Mosel, S. (2005). Self-directed learning with personal publishing and microcontent. In Microlearning 2005 conference, Innsbruck. https://bit.ly/3ejaC7B

Navarro, V., \& Di Bernardo, J. (2016). Una cápsula por semana: estrategia de Microlearning en una asignatura de Medicina. Revista de la Facultad de Medicina, 36(1), 40-42. https://revistas.unne. edu.ar/index.php/rem/article/view/2427/2141

Palazón, J. (2015). Aprendizaje móvil basado en microcontenidos como apoyo a la interpretación instrumental en el aula de música en secundaria. Píxel-Bit. Revista de Medios y Educación, 46, 119-136.

Pyc, M. A., \& Rawson, K. A. (2010). Why testing improves memory: Mediator effectiveness hypothesis. Science, 330(6002), 335-335. https://science.sciencemag.org/content/330/6002/335

Riveros, H. J. (2020). El reto de educar en tiempos de la digitalización de la vida: hacia una pedagogía de las relaciones entre cuerpo, texto y tecnología. Revista Boletín Redipe, Vol. 9, № 4, 2020, $90-$ 113. https://revista.redipe.org/index.php/1/article/view/951

Salinas, J., \& Marín, V. I. (2015). Pasado, presente y futuro del microlearning como estrategia para el REVISTA BOLETIN REDIPE 10 (2): $78-97$ - FEBRERO 2021 - ISSN $2256-1536$ 
desarrollo profesional. Campus Virtuales, 3(2), 46-61. https://bit.ly/3c4mZmH

Schlemmer, E., Zanela, A., Barbosa, J. \& Reinhard N. (2007). M-Learning ou aprendizagem com mobilidade: casos no contexto brasileiro. https://bit.ly/2B393wr

Trabaldo, S., Mendizábal, V. \& González, M. (2017). Microlearning: experiencias reales de aprendizaje personalizado, rápido y ubicuo. In IV Jornadas de TIC e Innovación en el Aula (La Plata). Recuperado de https://bit.ly/3glWw7t

Xue, G., Dong, Q., Chen, C., Lu, Z., Mumford, JA \& Poldrack, RA (2010). Greater Neural Pattern Similarity Across Repetitions Is Associated with Better Memory. Science, 330 (6000), 97-101.

Yin, R. (2003). Case Study Research. Design and Methods. Tercera Edición. Sage Publications.

Yunda, R. (2012). Capacitación Virtual: Uso de E-learning para la formación de los trabajadores en las Organizaciones (Tesis de licenciatura inédita). https://bit.ly/3emflp0 